\title{
Universality of Persistence Exponents in Two-Dimensional Ostwald Ripening
}

\author{
Jordi Soriano \\ Departament d'ECM, Facultat de Física, Universitat de Barcelona, Diagonal 647, E-08028 Barcelona, Spain
}

Ido Braslavsky and Di Xu

Department of Physics and Astronomy, Ohio University, Athens, Ohio 45701, USA

Oleg Krichevsky

Physics Department, Ben-Gurion University, Beer-Sheva 84105, Israel

Joel Stavans*

\begin{abstract}
Department of Physics of Complex Systems, Weizmann Institute of Science, Rehovot 76100, Israel
\end{abstract}
(Received 1 September 2009; published 24 November 2009)

\begin{abstract}
We measured persistence exponents $\theta(\phi)$ of Ostwald ripening in two dimensions, as a function of the area fraction $\phi$ occupied by coarsening domains. The values of $\theta(\phi)$ in two systems, succinonitrile and brine, quenched to their liquid-solid coexistence region, compare well with one another, providing compelling evidence for the universality of the one-parameter family of exponents. For small $\phi, \theta(\phi) \simeq$ $0.39 \phi$, as predicted by a model that assumes no correlations between evolving domains. These constitute the first measurements of persistence exponents in the case of phase transitions with a conserved order parameter.
\end{abstract}

DOI: 10.1103/PhysRevLett.103.226101

PACS numbers: $68.55 . \mathrm{A}-$, 05.40.-a, 64.75.St

When a homogeneous off-critical binary mixture is quenched deep enough, it separates into two phases, each characterized by a different concentration of the two constituents. The process of phase separation commences by the nucleation of droplets of one phase that grow both in size and number, until the droplet volume fraction $\phi$ nearly attains the equilibrium value determined by the lever rule. A late-stage regime then ensues whereby no more droplets form, and existing ones undergo a coarsening process in which large droplets grow at the expense of sufficiently small ones, with the average droplet size growing in time. This coarsening process, called Ostwald ripening, constitutes a classical example of the evolution of nonequilibrium, spatially extended systems [1]. The theoretical description of the late stage of this dynamics is a celebrated problem in statistical mechanics, starting from the seminal work of Lifshitz, Slyozov [2,3], and Wagner [4] (LSW). Assuming that the volume fraction of the coarsening phase is small $(\phi \rightarrow 0)$, so that domains evolve independently from one another, LSW predicted a scaling regime in which the average domain size increases as $t^{1 / 3}$, and the statistical pattern properties are time invariant.

The LSW exponent, being independent of $\phi$ and the same in two and three dimensions, cannot play a role classifying systems as critical exponents do in the case of equilibrium systems. Furthermore, it has become clear in recent years that characterizations such as two-time correlations do not capture fully the history of evolving, extended nonequilibrium patterns, and attention has focused on a new property, the persistence, that measures the statistics of first passage events in the system [5]. In general, persistence is the probability $P(t)$ that at a given point in space, a scalar order parameter has not crossed a threshold value up to time $t$, starting from a random initial configuration. In the scaling regime, $P(t)$ decreases algebraically at long times, $P(t) \sim t^{-\theta}$, where $\theta$ is the so-called persistence exponent. This exponent is nontrivial and unrelated to the two known static critical exponents and the dynamic exponent $z$ [5]. The average length of the longest excursions together with persistence exponents provide a good characterization of generic nonequilibrium dynamics [6].

Persistence exponents have been measured in various systems ranging from breath figures [7], one-dimensional gas diffusion [8], step fluctuations in crystals [9,10], coarsening two-dimensional soap froths [11], and advancing combustion fronts [12]. Of particular fundamental importance are evolving patterns produced as a result of phase transitions. Measurements of persistence exponents in thin layers of twisted nematic liquid crystal, which provide a physical realization of a quenched thermodynamic system characterized by an Ising-like nonconserved order parameter have been carried out [13]. However, beyond computer simulations of Ising ferromagnets with conserved Kawasaki-like dynamics [14], no measurements of persistence were performed in coarsening systems characterized by conserved dynamics and controllable volume fraction [15].

In a spatially extended coarsening system that has reached the late-stage regime, persistence has the natural interpretation of being the fraction of space that remains in the same phase after the initial quench. Lee and Rutenberg 
have calculated persistence exponents for Ostwald ripening in the limit of small values of $\phi$, for which local correlations in both growth rates and sizes can be neglected [16-18]. They found that $\theta$ is proportional to the area fraction, $\theta=\gamma_{2} \phi, \gamma_{2}=0.39008$ being a universal constant in two dimensions that is independent of quench depth, surface tension, temperature or mobility [15]. The analogous universal constant in three dimensions is different $\left(\gamma_{3}=0.50945\right)$.

Here we present experimental measurements of the persistence exponent $\theta$ in two-dimensional Ostwald ripening, as a function of the area fraction $\phi$. The integral of the density over the system's volume is the conserved parameter in this system. In an attempt to demonstrate the universality and utility of the concept of persistence, we have measured $\theta(\phi)$ in two different physical systems: thin layers of succinonitrile in their liquid-solid coexistence region [16,17], and the coarsening of ice crystals [19] in thin brine layers, as illustrated in Fig. 1. In both systems the average solid domain size increases as $t^{1 / 3}$, as predicted by the LSW model, irrespective of the volume (area) fraction occupied by solid domains However, there are significant $\phi$-dependent deviations in the distribution of domain sizes from the LSW prediction [16-18], which is only appropriate in the limit $\phi \rightarrow 0^{+}$. These deviations stem from correlations in sizes [20,21] (direct correlation effect) and rates of growth [22] (medium polarization effect) of neigh-
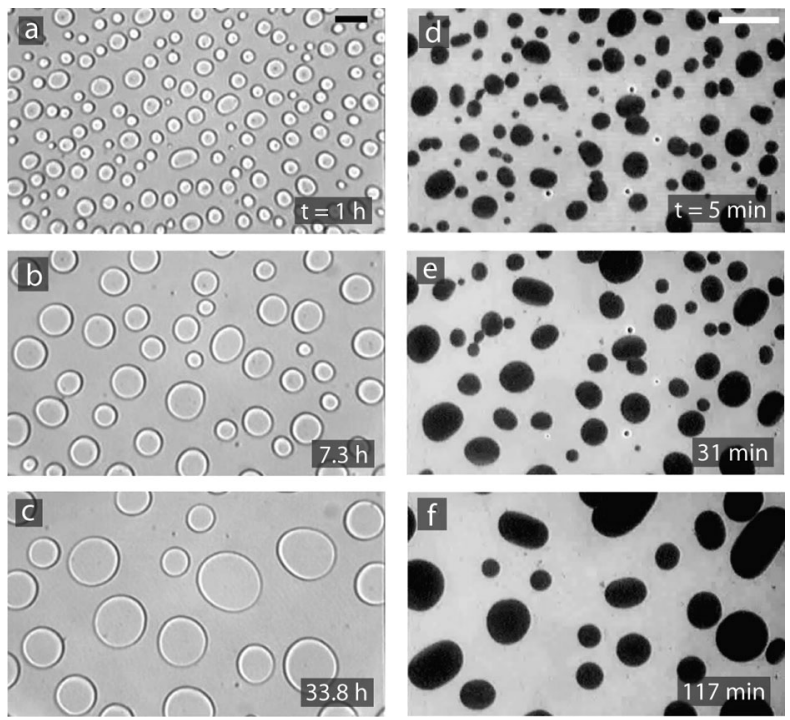

FIG. 1. Snapshots of coarsening solid domains in thin layers of succinonitrile and brine in the scaling state. (a)-(c) Succinonitrile quenched to $T=54{ }^{\circ} \mathrm{C}$, with $\phi=0.31$. (d)-(f) Ice quenched to $T=-0.68{ }^{\circ} \mathrm{C}$, with $\phi=0.25$. For both cases the thickness of the layer is $25 \mu \mathrm{m}$. The capture times indicate the time spanned since the beginning of recording, with the top snapshots (a) and (d) corresponding to the earliest detection of Ostwald's dynamics. Scale bars are $200 \mu \mathrm{m}$. In both systems the sequences of pictures cover a range of about a decade in solid domain size and two decades in time. boring domains. Correlations are already apparent for area fractions of few percents [18]. As we will show, our experimental results provide compelling evidence for the universality of $\theta(\phi)$, and in the small $\phi$ limit agree very well with the theoretical predictions of Lee and Rutenberg.

Experimental setup and procedure.-Experiments with succinonitrile were described extensively before [17]. Experiments on ice were performed on a cold stage described previously [23], whose temperature was controlled with a stability of $0.001{ }^{\circ} \mathrm{C}$. The sample cell consisted of a $3 \mu \mathrm{l}$ solution $(200 \mathrm{mM} \mathrm{NaCl}$ and $5 \mathrm{mM}$ fluorescein in double-distilled water) placed between two $12 \mathrm{~mm}$ diagonal glass cover slips. The calculated height of the liquid was about $25 \mu \mathrm{m}$. The sample cell was sealed with oil (Type DF, Cargille, Cedar Grove, NJ) to avoid evaporation of the water.

The cover-slip cell was placed in thermal contact between two copper plates to ensure low temperature gradients in the sample, one of which contained a $2 \mathrm{~mm}$ diameter hole for observation covered with a third cover slip as window for observation. This multilayer arrangement was placed on the cold stage within a thermally anchored metal box. Dry air was continuously circulated through the box to avoid fog on the observation window.

The experimental stage was placed on a fluorescence microscope (BH2, Olympus, Center Valley, PA) equipped with a fluorescence cube suitable for fluorescein observation, and illumination was modulated with a computercontrolled shutter (Model 225, Uniblitz, Rochester, NY). Ice rejects both salt and fluorescein and thus could be clearly detected with fluorescence imaging as dark patches in a bright background as illustrated in the right panels of Fig. 1. We used a $4 \mathrm{X}$ objective to image the sample. A CCD camera (SPT-M124, Sony, Park Ridge, New Jersey) was used to record images directly to a computer via a Labview-controlled video frame grabber (IMAQ-PCI1407, National Instruments). Images were taken every minute with an integration time of $2 \mathrm{~s}$ in order to reduce the bleaching of the dye. Typical runs lasted for $6 \mathrm{~h}$. The LABVIEW program controlled the temperature of the sample, and also calculated $\phi$ in real time.

A sample was initially cooled to a temperature between -15 and $-20^{\circ} \mathrm{C}$, freezing the liquid completely. The sample was then warmed until part of the ice melted and fragmented into small grains. In order to overcome residual concentration gradients and the effect of finite cell size, the temperature was slowly adjusted (at a maximum rate of $0.002{ }^{\circ} \mathrm{C} / \mathrm{min}$ ) to keep $\phi$ constant.

Ice is a uniaxial material with a strong anisotropy in many parameters. In particular, for the small supercoolings which were used in these experiments, ice grows slowly and in a faceted manner in the $c$ axis direction and faster with no faceting in the perpendicular direction to the $c$ axis. In a confined nearly two-dimensional $\sim 25 \mu$ m-high cell, crystals whose $c$ axis was perpendicular to the sample 
plane, a circular crystal shape resulted whereas crystals whose $c$ axis was parallel to the sample plane resulted in rectangular shapes. Raising the temperature in a controlled fashion, individual crystals could be partially melted, becoming smaller than $25 \mu \mathrm{m}$ and thus allowing them to rotate. Upon growth (as disks), the glass walls aligned crystals so that to have their $c$ axis perpendicular to the sample plane.

Image and data analysis.-From each experiment with either succinonitrile or ice a family of about 20 images were extracted, and covering the entire duration of the experiment in logarithmic scale in time. Each image was processed and thresholded to obtain a sharp, black and white description of the evolving system. The images were then studied to extract the time evolution of the growing domains and verify that their average radii obeyed the scaling regime $r(t) \sim t^{1 / 3}$. An image at time $t_{0}$ within the scaling regime was then chosen and used as the base image to overlay all subsequent, thresholded images up to time $t$.

The resulting composition provided the unswept area $A(\tau)$ that was never visited by a domain, with $\tau=t-t_{0}$. The persistence exponent $\theta$ was determined as a power law fit $A(\tau) \sim \tau^{-\theta}$ at long $\tau$. We repeated the analysis using different base images $t_{0}$ and considering different power law fitting intervals. The value of $\theta$ that we finally used is the average over the different analyses, and their corresponding error bars take into account both the spread of values in $\theta$ and the artifacts from image processing and thresholding.

Results and discussion.-To compute the persistence exponent in our experiments for given values of $\phi$, the area $A(t)$ unswept by domain boundaries up to time $t$ after the quench was measured from a library of pictures obtained during coarsening, as is illustrated in Fig. 2.
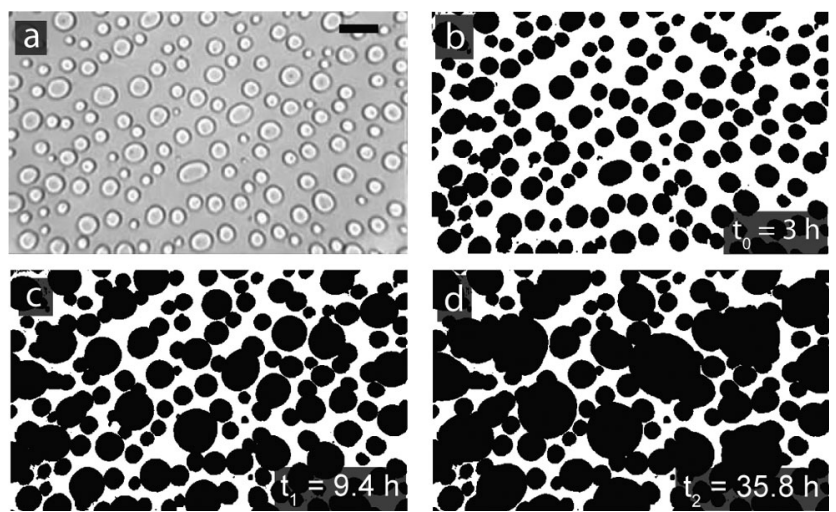

FIG. 2. Algorithm for the calculation of persistence exponents. (a) Typical picture of coarsening domains of succinonitrile at $\phi=0.31$. (b) Same snapshot as in (a) after thresholding, used as base image $t_{0}$. (c) Superposition, over image at time $t_{0}$, of all pictures obtained up to time $t_{1}$ after thresholding. (d) Same as in (c) but up to a later time $t_{2}\left(t_{1}<t_{2}\right)$. Scale bar is $200 \mu \mathrm{m}$.
We show a typical early-time image of coarsening succinonitrile crystals obtained at $\phi=0.31$ in Fig. 2(a) and the same image after applying a threshold in Fig. 2(b), in order to highlight the solid domains. After applying the threshold filter to the full library, all pictures corresponding to times prior to $t$ were overlaid, as shown for intermediate and late times $t$ in Figs. 2(c) and 2(d), respectively, and the white area $A(t)$ was measured. For late times $t, A(t)$ decays as a power law with the persistence exponent $A(t) \sim t^{-\theta}$. In Figs. 3(a) and 3(b) we show typical plots of $A(t)$ normalized by the total area for both succinonitrile and ice crys-
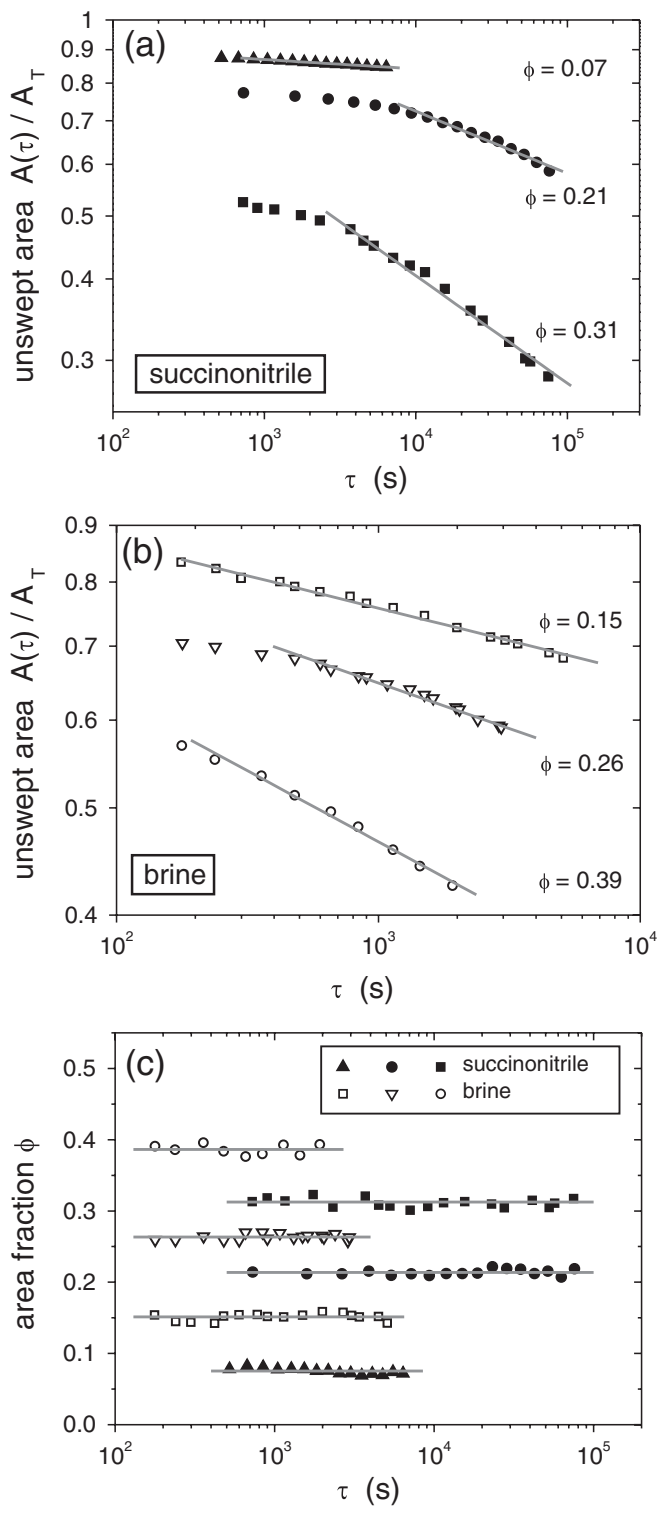

FIG. 3. Area $A(t)$ unswept by domain boundaries up to time $t$, normalized by total system area. (a) Typical results for succinonitrile obtained at various area fractions $\phi$. (b) Similar data as for the top but for ice crystals in brine. Solid lines in (a) and (b) are fits to the long time regime of the data, whose slope yields the persistence exponent $\theta$. (c) Constancy of $\phi$ throughout time; lines are the average value of $\phi(\tau)$. 


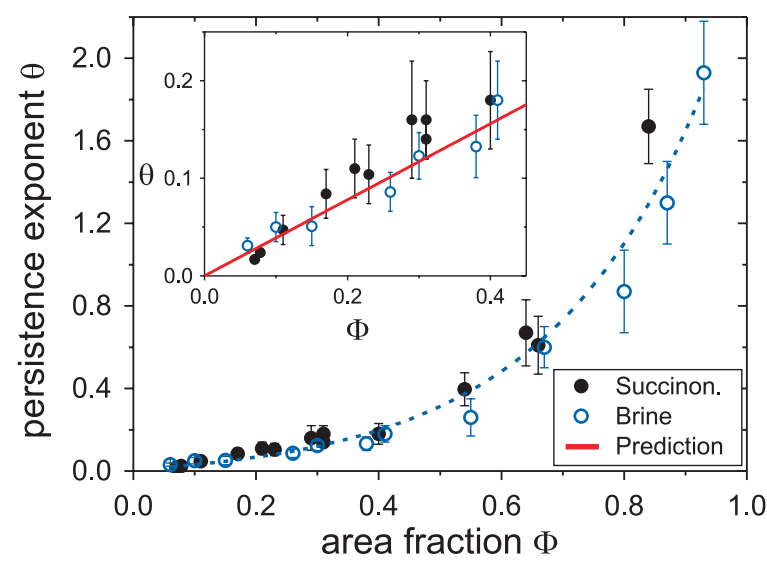

FIG. 4 (color). One-parameter family of persistence exponents $\theta$ parametrized by the area fraction $\phi$. Solid circles correspond to succinonitrile data while empty circles to ice. The dashed line is a guide to the eye. Inset: detail of the small- $\phi$ region of the data. The red line is the small- $\phi$ theoretical prediction $\theta=$ $0.39008 \phi$ by Lee and Rutenberg [15]. Error bars reflect both the freedom in the choice of range over which the fits to determine $\theta$ were carried out (e.g., Fig. 3), as well as the dependence of the value of $\theta$ on the choice of first image such as that shown in Fig. 2(a).

talline domains. The area fraction $\phi$ remains practically constant during the measurements, as shown in Fig. 3(c).

The central result of this work is presented in Fig. 4, where we show a plot of $\theta(\phi)$ for both succinonitrile solid domains and ice crystals in brine. Both systems yield comparable values of $\theta(\phi)$ that increase linearly with $\phi$ to a good approximation, as long as $\phi$ remains small. For intermediate and large values of $\phi$, however, i.e., deeper quenches, there is an increasing deviation from linearity, and $\theta$ reaches large values, reflecting a faster rate of memory loss of initial conditions. We ascribe the deviation from linearity to correlations in growth. Indeed, correlations, which become stronger with $\phi$, can induce domain distortions whose effect is to reduce $A(t)$ (see, for example, Fig. 10 in Ref. [17]). We now compare the prediction of Lee and Rutenberg with our data. As shown in the inset of Fig. 4, their prediction compares very favorably with experiment, the exponents in both experiments approaching the linear dependence asymptotically $\left(\phi \rightarrow 0^{+}\right)$. Indeed, linear fits to both succinonitrile and ice data, in the range $0 \leq \phi<0.45$, yield $0.43 \pm 0.04$ and $0.39 \pm 0.03$ for $\gamma_{2}$, respectively.

Despite considerable differences between the two systems such as temperature and composition, the families of persistence exponents $\theta(\phi)$ measured in our experiments coincided within experimental error, providing strong evidence for the universality of $\theta(\phi)$. This finding clearly indicates that persistence exponents may play a role characterizing nonequilibrium systems analogous to the one critical exponents play in the case of equilibrium. It remains as a future theoretical challenge to include correlations between growing domains in calculations of $\theta(\phi)$.

J. Stavans thanks S. Majumdar for conversations that inspired the work presented here, and M. Evans for hospitality. I. B. acknowledges support by the National Science Foundation under Grant No. CHE-0848081 (cofunded by the MPS/CHE and the Molecular and Cellular Biosciences Divisions, and by the Office of International Science and Engineering and the Office of Polar Programs).

*Joel.Stavans@weizmann.ac.il.

[1] A. Bray, Adv. Phys. 43, 357 (1994).

[2] I. M. Lifshitz and V. V. Slyozov, Zh. Eksp. Teor. Fiz. 35, 479 (1958).

[3] I. M. Lifshitz and V. V. Slyozov, J. Phys. Chem. Solids 19, 35 (1961).

[4] C.Z. Wagner, Z. Elektrochem. 65, 581 (1961).

[5] S. N. Majumdar, Curr. Sci. 77, 370 (1999).

[6] C. Godrèche, S. N. Majumdar, and G. Schehr, Phys. Rev. Lett. 102, 240602 (2009).

[7] M. Marcos-Martin et al., Physica (Amsterdam) 214A, 396 (1995).

[8] G. P. Wong et al., Phys. Rev. Lett. 86, 4156 (2001).

[9] M. Constantin et al., Phys. Rev. Lett. 91, 086103 (2003).

[10] M. Constantin et al., J. Stat. Mech. (2007) P07011.

[11] W. Y. Tam et al., Phys. Rev. Lett. 78, 1588 (1997).

[12] J. Merikoski et al., Phys. Rev. Lett. 90, 024501 (2003).

[13] B. Yurke et al., Phys. Rev. E 56, R40 (1997).

[14] P. Gonos and A. J. Bray, J. Phys. A 38, 1427 (2005).

[15] B. P. Lee and A. D. Rutenberg, Phys. Rev. Lett. 79, 4842 (1997).

[16] O. Krichevsky and J. Stavans, Phys. Rev. Lett. 70, 1473 (1993).

[17] O. Krichevsky and J. Stavans, Phys. Rev. E 52, 1818 (1995).

[18] B. Levitan and E. Domany, Phys. Rev. E 57, 1895 (1998).

[19] T. Hagiwara et al., Food Biophys. 1, 74 (2006).

[20] M. Tokuyama and K. Kawasaki, Physica (Amsterdam) 123A, 386 (1984).

[21] M. Marder, Phys. Rev. Lett. 55, 2953 (1985).

[22] J. A. Marqusee and J. Ross, J. Chem. Phys. 80, 536 (1984).

[23] N. Pertaya et al., Biophys. J. 92, 3663 (2007). 